Meta

Journal des traducteurs

Translators' Journal

\title{
Les comparaisons linguistiques et la comparaison bilingue intégrale
}

\section{Rostislav Kocourek}

Volume 33, numéro 4, décembre 1988

Symposium AILA 1987, Sydney

URI : https://id.erudit.org/iderudit/003332ar

DOI : https://doi.org/10.7202/003332ar

Aller au sommaire du numéro

Éditeur(s)

Les Presses de l'Université de Montréal

ISSN

0026-0452 (imprimé)

1492-1421 (numérique)

Découvrir la revue

Citer cet article

Kocourek, R. (1988). Les comparaisons linguistiques et la comparaison bilingue intégrale. Meta, 33(4), 542-549. https://doi.org/10.7202/003332ar d'utilisation que vous pouvez consulter en ligne.

https://apropos.erudit.org/fr/usagers/politique-dutilisation/ 


\title{
LES COMPARAISONS LINGUISTIQUES ET LA COMPARAISON BILINGUE INTÉGRALE
}

\author{
ROSTISLAV KOCOUREK \\ Université Dalhousie, \\ Halifax, Nouvelle-Écosse, Canada
}

La grande énigme des langues, c'est leur nombre et leur diversité, leur origine et leur avenir. $Y$ avait-il différenciation à partir d'une seule langue mère, comme le croyaient les partisans de la monogenèse ? Y aura-t-il réduction du nombre des langues et convergence vers une langue commune unique, comme le prétendait un autre extrême de la pensée linguistique ? Y aura-t-il, enfin, préservation de la diversité actuelle ? Toujours est-il qu'il existe aujourd'hui des milliers de langues, dont des centaines sont très bien décrites et cultivées, chacune avec plusieurs millions de locuteurs.

Parler de la diversité des langues suggère déjà la possibilité de leur comparaison. Les Grecs anciens ne se sont guère intéressés aux langues des barbares, mais les Romains observaient leur langue latine parallèlement à la langue grecque (par exemple le grammairien Didymus). Le Moyen Âge européen contemplait, à son tour, les langues à travers le prisme du latin (par exemple AElfric Grammaticus). C'est ainsi qu'a été établie la tradition d'une comparaison bilingue.

La grande époque de la comparaison multilingue n'a vraiment commencé qu'au XIXe siècle, une génération après la célèbre conférence de Sir William Jones à Calcutta le 2 février 1786 - dont nous venons de célébrer le bicentenaire -, ou, si l'on préfère, après l'important compte rendu de Christian Jakob Kraus, publié en 1787 (cf. Arens 1969 : 138-146 et Mounin 1967: 156-157).

Comparaison n'est pas raison mais les deux vont très bien ensemble. C'est ce que comprenait William Blake $(\dagger 1827)$, qui les rejetait toutes les deux, préférant la création : "I will not Reason and Compare : my business is to Create". Mais, ne peut-on pas créer en comparant?

La comparaison est un procédé de réflexion et d'imagination par excellence. " Il n'y a rien que l'esprit humain fasse si souvent que des comparaisons ", dit l'Encyclopédie de 1751-1780 (Diderot et d'Alembert $1966:$ t. 3, 745). La comparaison n'est pas seulement fréquente, elle est essentielle pour la pensée savante. "Comme nous ne savons rien que par comparaison, dès que tout rapport nous manque, et qu'aucune analogie ne se présente, toute lumière fuit » (le Robert 1966 : vol. 1, 850). C'est ce que pensait Buffon $(\dagger 1788)$, le Pline français de Montbard en Bourgogne dont les idées ont grandement influencé la grammaire comparée.

L'un des dictionnaires contemporains de la terminologie philosophique soutient encore aujourd'hui que "la comparaison est l'essentiel de toute étude scientifique " et qu'elle " suit description et précède explication " (Foulquié 1969 : 106). Mais la description dépend déjà de la comparaison. Ne présuppose-t-elle pas la définition des termes descriptifs, et la définition n'est-elle pas basée sur la comparaison? 
On peut en effet parler de l'ubiquité de la comparaison dans un discours savant, et les chercheurs essaient de préciser et de circonscrire cette notion très large, dont nous écartons ici le sens littéraire et le sens étroitement grammatical.

On fera grâce au lecteur de l'analyse sémantique que nous avons faite d'une dizaine de définitions disponibles et qui a donné la définition synthétique suivante : la comparaison est une recherche et un examen des rapports entre plusieurs choses différentes liées toutefois par certains caractères semblables. Cette définition nous servira de départ pour l'analyse des comparaisons linguistiques.

Les CHOSES comparées de la comparaison linguistique sont divers faits de langue. La comparaison concerne toute réflexion linguistique : définir un phonème, indiquer la fonction d'un morphème, décrire un temps verbal, déterminer la structure profonde, découper une unité lexicale, constater la synonymie - autant d'actes de comparaison linguistique.

Roman Jakobson (1963: 80) note que " l'équivalence dans la différence est le problème cardinal du langage et le principal objet de la linguistique ». Il est évident que non seulement rapprocher les choses différentes mais également distinguer les choses similaires sont des objets essentiels d'un domaine de connaissance tel que la linguistique. même.

La complexité des comparaisons possibles reflète la complexité de la langue elle-

On compare les traits pertinents, même non pertinents, les phonèmes, les graphèmes, les morphèmes, les mots, les syntagmes lexicaux et libres, les propositions, les phrases, les textes.

On compare la forme, la structure, le sens, la fonction.

On apparie les occurrences, les variantes, les unités abstraites.

On rapproche deux unités, deux catégories, deux sous-systèmes, deux systèmes (phonèmes; phonologie; langue tout entière).

On compare les faits de langue dont la variation est géographique, historique, sociale, stylistique, fonctionnelle.

Une distinction fondamentale est celle qui est faite entre la comparaison des aspects de la même langue (comparaison intralinguistique), et la comparaison de plusieurs langues différentes (comparaison interlinguistique, plurilingue, des langues). Dans ce qui suit, on va se concentrer sur la comparaison des langues.

En ce qui concerne le NOMBRE des langues comparées, certains domaines comparatifs sont généralement plurilingues, ou plutôt multilingues. Les titres étendus de delux célèbres grammaires comparées du XIXe siècle nomment chacun neuf langues : Vergleichende Grammatik de Franz Bopp (1 ${ }^{\text {re }}$ éd. 1833-1852) et Compendium der vergleichenden Grammatik der indogermanischen Sprachen (1 $1^{\text {re }}$ é. 1861-1862) de August Schleicher. L'index de l'Introduction à l'étude comparative des langues indoeuropéennes (1903) d'Antoine Meillet comprend plus de 80 langues, dont 43 apparaissent assez souvent pour mériter une abréviation.

Mais la structure linguistique étant fort complexe, on suppose qu'il est raisonnable de limiter le nombre des langues comparées à deux, et d'arriver, si nécessaire, aux cornparaisons multilingues par le chemin sûr de la comparaison à deux termes qui exige "une double attention " pour citer Condillac (cf. Lalande 1951 : 154). La comparaison bilingue a une longue tradition. C'est elle qui constitue le noyau de nos réflexions.

Si l'on prétend que la comparaison est une RECHERCHE de rapports, on présuppose d'abord que de tels rapports existent et que l'on possède les connaissances nécessaires pour les trouver. La découverte des rapports n'est pas automatique, les rapports essentiels sont souvent cachés ou non existants. Le succès est donc contingent. En plus, certains rapports signalés sont si éloignés des données primaires que leur existence 
ne peut être ni confirmée ni infirmée ; il se peut que certains rapports soient plutôt inventés que découverts.

La découverte des rapports est unie à leur EXAMEN, une opération mentale volontaire, intellectuelle, cognitive, méthodique. L'examen comparatif bilingue peut être neutre (réciproque) ou orienté (directionnel). Il est neutre s'il essaie de relever les ressemblances et les différences du point de vue des deux langues ; par exemple le manuel Stylistique comparée est considéré comme tel par Alfred Malblanc (Vinay et Darbelnet $1968: 2$ ). L'examen est orienté si une langue sert de toile de fond (langue de départ, thématique) pour la caractéristique de l'autre langue (langue d'arrivée, rhématique). Dans ce dernier cas, il y a en principe deux façons différentes de comparer la même paire de langues, selon que l'une ou l'autre est langue rhématique. Dans sa Linguistique générale et linguistique française, Charles Bally se proposait « d'éclaircir le visage du français par la comparaison de l'allemand " (Bally 1950:29).

Examinons maintenant le problème des RAPPORTS de différence et de ressemblance. Pour que l'on puisse comparer deux langues, il faut sans doute qu'elles soient à la fois différentes et similaires. Quant à l'importance relative des différences et des ressemblances, les positions théoriques et fonctionnelles oscillent entre deux extrêmes.

D'abord la position qui souligne les différences. Réagissant contre les excès du comparatisme du XIXe siècle, certains linguistes maintiennent que toutes les langues sont uniques, que - hors du système où tout se tient - il n'y a pas d'équivalence, pas d'opposition, pas de rapport pertinent. Ce postulat de l'unicité de chaque langue dégage des forces de l'analyste et permet une description poussée de la langue donnée. Mais, un peu comme les critiques littéraires qui n'analysent et ne connaissent que leur auteur préféré, cette approche a des limites importantes. La comparaison des langues est très coincée dans l'atmosphère bornée de cette conception particularisante.

Si les particularistes sous-estiment la comparaison parce qu'ils s'intéressent surtout à la structure individuelle de chaque langue, les universalistes sont fascinés par les ressemblances. Roger Bacon (†1294), Doctor Mirabilis du Moyen Âge, Oxonien et Parisien, représente déjà cette exigence de l'universalité grammaticale, chère à son époque, qu'il a formulée d'une façon admirablement concise : "Grammatica una et eadem est secundum substantiam, licet accidentaliter varietur " (Robins $1967: 77,91$ ), c'est-à-dire "La grammaire est une et la même selon la substance, quoiqu'elle varie dans les accidents 》 (attention à la traduction française dans Robins $1976: 81$ ). On voit que les différences sont reléguées au second plan, dans une subordonnée concessive (licet).

Plus récemment, en 1958, Roman Jakobson (1963:68) maintient qu'il n'y a pas de différence de principe entre les systèmes linguistiques du monde. La comparaison et la caractérisation des langues se trouvent parfois marginalisées par les théories qui mettent plus d'accent sur la généralité de la langue naturelle que sur les particularités des langues individuelles.

Ce qu'il faut c'est un point de vue équilibré. Dire que toutes les langues sont égales est tautologique. C'est dire seulement que toute langue est une langue (on ne parle pas ici de droits linguistiques des locuteurs des langues données). On serait tenté d'ajouter une restriction orwellienne et de dire que toutes les langues sont égales, mais que certaines sont plus égales que d'autres. C'est-à-dire qu'il y a, quand même, des différences importantes.

Il en va de même pour les différences. Edward Sapir a écrit en 1921, à Ottawa d'ailleurs, que - et je traduis - " nous pouvons dire que toutes les langues sont différentes ". Il se dépêche cependant d'ajouter : " mais certaines diffèrent entre elles beau- 
coup plus que d'autres " (Sapir 1921: 121 ; attention à la traduction française dans Sapir 1953 : 115). Cela veut dire qu'il y a des ressemblances non négligeables.

En examinant les rapports, on se trouve devant nombre de problèmes théoriques, dont au moins deux dignes de mention en tant que problèmes fondamentaux. C'est, en premier lieu, l'ASYMETRIE STRUCTURELLE potentielle. Deux langues peuvent diverger d'une manière importante, remplissant les mêmes fonctions de deux façons très différentes. Ce qui est grammaticalisé dans une langue ne correspond qu'à des moyens d'équivalence contingents, non systématiques dans l'autre langue. Ce qui constitue un problème morphologique d'un côté peut être un problème syntaxique syntagmatique de l'autre. Un élément structuro-sémantique obligatoire d'une langue peut être complètement sans pertinence dans l'autre.

Le second problème se pose s'il existe un parallélisme structurel important ; c'est la construction de la BASE NOTIONNELLE COMMUNE, subsumant les deux faits de langue comparés. Comparer c'est analyser les catégories des deux langues en composantes, et construire, ou au moins présupposer, une catégorie superordonnée qui subsumerait les catégories comparées.

Si l'on décrit par exemple le nom français, la catégorie linguistique est donnée par la spécificité du français (nombre, genre, article, accord, par ex.). Si l'on compare le nom français au nom latin, anglais ou allemand, on a polysémie de nom. L'un des pièges de la comparaison est de s'imaginer que ressemblance soit identité. La ressemblance ne devient identité qu'au niveau abstrait d'une catégorie superordonnée : nom ${ }_{0}$ (superordonné), qui subsumerait nom 1 (latin), nom 2 (français), nom 3 (anglais), nom 4 (allemand), etc. $\mathrm{Nom}_{0}$ serait un hyperonyme dont l'extension serait supérieure et la compréhension inférieure à l'extension et à la compréhension des autres sens de nom.

Un effort théorique important est parfois nécessaire pour permettre des comparaisons systématiques. Dans le même ordre d'idées, on peut nommer les tentatives de comparer la langue naturelle avec d'autres systèmes de signification qui ont mené à l'élaboration d'une théorie sémiotique dont les catégories sont supposées superordonnées aux caltégories linguistiques qu'elles subsument.

Quels domaines de la linguistique peuvent être considérés comme APPROCHES de la comparaison des langues? Nous ne présenterons pas une taxinomie artificiellement construite de types hypothétiques (cf. Ellis 1966). Nous signalerons les approches disponibles telles qu'elles se sont développées en linguistique. Leur variété est surprenante.

Commençons par la comparaison GÉNÉTIQUE (ou généalogique), qui est la fierté de la linguistique de tout temps. Elle a cherché, trouvé et développé des méthodes et des critères applicables à la découverte et à l'examen des concordances entre langues parentes, et à leur évolution. Il s'agit d'une comparaison multilingue, diachronique et orientée vers le passé, à la recherche d'une ancienne langue commune. Les comparatistes génétiques - et leurs critiques - ont aidé à constituer les fondements de la grammaire comparée multilingue, mais aussi de la grammaire historique unilingue et de l'étymologie.

Les comparatistes, tel Herman Paul $(† 1921)$, le codificateur de la théorie des néogrammairiens, étaient convaincus que leur approche historique était la seule méthode savante possible dans les recherches linguistiques. "La seule classification linguistique qui ait une valeur et une utilité est la classification généalogique fondée sur l'histoire des langues " - c'est ce que croit le comparatiste français Antoine Meillet (†1936) et encore en 1924 (Meillet 1952 : 53).

Malgré cette opinion très répandue à l'époque, on doit noter que la comparaison, en tant que telle, est neutre par rapport au temps (achronique, selon Greimas et Courtés 
1979 : 49). Parmi toutes les approches de la comparaison, la comparaison génétique bien que la mieux développée - est la seule à être exclusivement fondée sur une perspective historique.

La hantise du passé, du changement et de la parenté génétique s'oppose à une comparaison ou à une classification des faits de langue inspirée surtout par Wilhelm von Humboldt $(\uparrow 1835)$ et nommée TYPOLOGIQUE. La comparaison typologique est une comparaison multilingue, orientée vers la classification non génétique des langues.

En principe, la comparaison typologique vise la totalité des langues, mais elle est normalement restreinte à certains groupes de langues, et n'observe qu'un nombre limité de traits typologiques, choisis arbitrairement. Parmi les difficultés de cette approche est l'inévitable non coïncidence des résultats aux niveaux différents. Par exemple, les mêmes langues ne se regroupent pas nécessairement de la même façon au niveau phonologique et au niveau grammatical ou lexical.

Les traits typologiques ne sont pas des traits communs à toutes les langues. Ils ne comprennent donc pas les traits universels que l'on nomme universaux linguistiques (syn. universaux des langues naturelles dans Greimas et Courtés 1979:410, et universaux du langage dans Dubois et al. $1973: 503$ ). Étant donné la diversité des langues, la difficulté de leur saisie coïncidente, et le manque d'un système notionnel et terminologique suffisamment général, la détermination satisfaisante des universaux linguistiques semble à peine possible. Leur recherche pose toutefois des questions fondamentales dont la discussion ne peut qu'approfondir la réflexion linguistique à penchant logicosémantique et philosophique.

Il est évident que la recherche des universaux linguistiques est l'approche la plus générale de la comparaison des langues. Nous l'appellerons comparaison UNIVERSELLE (comparaison à base d'universaux). C'est une comparaison ahistorique positive, orientée vers les concordances.

Ni la généralité des comparaisons typologique et universelle, ni l'historicisme de la comparaison génétique ne permettent la saisie détaillée et profonde des rapports linguistiques structurels qui existent entre les systèmes de deux langues données. Il est donc important de disposer d'une comparaison de langues qui serait systémique et agénétique, bilingue ou paucilingue, et qui tâcherait de confronter tous les aspects essentiels des langues observées. Ce sont surtout ces rapports-là qui influencent, intéressent, inspirent, déroutent les utilisateurs des deux langues.

Il est intéressant de noter le silence que l'on garde sur le fait que les tentatives d'une telle comparaison ont une tradition plus longue que la comparaison génétique. Il y a par exemple presque seize cents ans, vers 400 ap. J.-C., que le grammairien latin Macrobius mettait en relief les différences et les concordances (lat. societates) du verbe grec et du verbe latin (cf. Keil éd. 1855-1880: v. 5, 595-655). Depuis Macrobius, on n'a jamais complètement abandonné la comparaison bilingue de la langue maternelle et l'autre langue principale d'un pays et d'une époque donnés.

$\mathrm{Au} \mathrm{XX}^{\mathrm{e}}$ siècle, une approche structurelle a reçu l'attention théorique. Réagissant contre un génétisme exagéré, les écoles de Prague et de Genève ont le mérite d'avoir posé la question de ce genre de comparaison de langues.

En 1929, le regretté Bohumil Trnka (†1984) expliquait non seulement l'importance d'une comparaison non génétique, qu'il appelait analytique, mais aussi - très lucidement - le caractère complémentaire de la comparaison analytique et génétique (Trnka $1982: 23-28$ ).

En 1932, Charles Bally ( $† 1947)$ appelait statique le point de vue qui consiste à relever " dans les deux langues, surprises à un moment précis de leur histoire, sans souci de prouver une parenté généalogique, des caractères différentiels qui accusent plus for- 
tement l'originalité de chacune d'elles et le type général dont chacune relève " (Bally $1950: 28)$.

Je propose d'utiliser, pour cette comparaison, le terme comparaison STRUCTURELLE. Sans être idéal, le modificateur structurel paraît préférable aux adjectifs comme analytique, contrastif, synchronique, statique, systémique. La comparaison structurelle est une approche qui se veut savante et désintéressée (non appliquée mais applicable), neutre plutôt qu'orientée. C'est la comparaison agénétique principale, qui devrait être capable de saisir toutes les données des rapports agénétiques.

Aucune des approches précédentes n'est faite sur mesure pour répondre aux besoins de la didactique des langues étrangères. La comparaison génétique est passionnante pour les spécialistes et pour les polyglottes, mais ses résultats n'ont aujourd'hui qu'une application didactique restreinte. Le rôle, dans l'enseignement, des comparaisons typologique et universelle est marginal. Et la comparaison structurelle, peu sensible aux besoins précis des apprenants et des enseignants, s'est vue remplacée par ce qu'on appelle la linguistique contrastive (Fisiak éd. 1981) ou différentielle. Nous parlerons de la comparaison CONTRASTIVE.

Qu'elle soit pratique ou théorique, la comparaison contrastive est didactique, habituellement bilingue, synchronique contemporaine, orientée vers la langue seconde comme langue cible (rhématique), basée sur la langue maternelle comme langue de départ (thématique), avec un accent sur des différences de structure. La comparaison contrastive est très liée à la comparaison structurelle, mais sa sélectivité et son pragmatisme programmatiques la distingueront toujours des analyses linguistiques plus désintéressées et intégrales, et moins ponctuelles.

Proche de la comparaison contrastive est la comparaison qui examine les aspects linguistiques de l'influence d'une une langue sur l'autre. Nous proposons de l'appeler comparaison INTERFÉRENTIELLE, au sens linguistique, et non psychologique ou pédagogique. Elle regroupe les problèmes linguistiques du bilinguisme ou du multilinguisme, des langues en contact et de l'emprunt (cf. Weinreich 1968 et 1970, et Deroy 1956). Cette étude, qui peut être diachronique ou synchronique, est motivée par ses corrélations socioculturelles ou ethnologiques plutôt que didactiques. Elle est orientée vers la langue subissant l'interférence.

Aucune des définitions de la comparaison que nous avons observées ne comprend l'aspect de qualité. Mais ne compare-t-on pas souvent afin de décider laquelle des deux choses est meilleure par rapport à certains critères ? On voit bien les dangers, les pièges et parfois l'impossibilité de l'évaluation objective des langues (cf. Martinet 1969: 46-61 ; Kukenheim 1966 : 155). Mais la question ne disparaitra pas parce qu'on l'aura chassée (cf. Jespersen 1969 : 29-31, 99). Il existe donc une comparaison ÉVALUATIVE en linguistique, bien qu'elle ait été maniée d'une façon peu encourageante dans le passé, quand il s'agissait, par exemple, de la recherche d'une langue universelle, idéale ou auxiliaire. (Pour l'évaluation en terminologie, v. Kocourek 1985.)

La faiblesse troublante de cette approche est, cependant, qu'il est difficile, sinon impossible, d'évaluer sa propre langue maternelle objectivement par rapport aux langues secondes, non maternelles. C'est pourquoi nous laisserons en marge ce type de comparaison intéressant mais hasardeux.

Les deux dernières approches comparatives - lexicographie et traduction - se distinguent des sept approches précédentes par des niveaux de généralisation moins élevés. Souvent négligés dans les études comparatives théoriques, ces deux types représentent un trésor de données comparatives de base.

D'abord la lexicographie bilingue. Le dictionnaire bilingue, probablement l'outil le plus populaire des utilisateurs des langues secondes, est un produit de la comparaison 
bilingue que nous appellerons comparaison LEXICOGRAPHIQUE. Le plus souvent, il s'agit d'une comparaison bilingue, synchronique, lexico-sémantique, de généralisation restreinte. La généralisation dépasse le niveau de l'occurrence mais elle s'arrête au niveau de l'unité lexicale. Les données présentées, dont le but immédiat est de faciliter la compréhension et la traduction, peuvent en plus servir, d'une part, de source importante d'une théorie de lexicographie bilingue, et, d'autre part, pour l'élaboration d'une comparaison lexico-sémantique structurelle des deux langues.

À la différence de la comparaison lexicographique, qui cherche surtout l'équivalence paradigmatique des unités lexicales et de leurs acceptions variées, le type suivant consiste à chercher l'équivalence des textes ; il s'agit de la traduction, dont l'aspect principal nous appellerons comparaison TRADUCTRICE.

Si la traduction est l'expression d'un texte source par un texte cible équivalent, la recherche de l'équivalence textuelle consiste à identifier tous les traits pertinents du texte en langue source, à chercher les traits correspondants en langue cible et à produire un texte équivalent (cf. Kocourek 1982 : 181). Pour ce faire, il faut tenir compte de tous les moyens de la langue qui sont employés dans le texte source et que l'on doit reconstituer par le texte cible. La comparaison traductrice est donc ponctuelle, occurrentielle, elle n'est structurelle qu'indirectement. Elle ne compare les deux systèmes linguistiques que d'une manière sélective, étant limitée aux traits traductionnellement pertinents du texte source et à leurs équivalents en langue cible.

La comparaison traductrice est bilingue, textuelle, orientée vers le texte en langue cible. La généralisation s'arrête au niveau d'un texte individuel. Mais les textes appariés source-cible comprennent une richesse inépuisable de données primaires pour l'élaboration d'une théorie de traduction, et pour une comparaison structurelle des deux langues. Cette comparaison dépasse le niveau lexico-sémantique parce qu'elle traite également des rapports fonctionnels grammaticaux et stylistico-textuels.

Nous avons pu relever un nombre important d'approches disponibles de la comparaison des langues. C'est la comparaison génétique, et diverses approches agénétiques dont la comparaison typologique, universelle, structurelle, contrastive, interférentielle, évaluative, lexicographique et traductrice.

Chacune de ces neuf approches s'est développée d'une manière plus ou moins indépendante. Il est normal et souhaitable que ces approches continuent à le faire pour satisfaire aux besoins des intéressés.

On peut aussi, cependant, considérer toutes ces approches comme complémentaires pour arriver à une comparaison bilingue plus complète, INTÉGRALE . Cette comparaison intégrale peut profiter des résultats de tous les types nommés. Le niveau très général de la comparaison universelle représente une introduction au problème. La comparaison typologique constitue un cadre utile de première différenciation, elle détermine les caractéristiques générales de chacune des deux langues. Dans la mesure où il s'agit de langues parentes, la comparaison génétique découvre les rapports génétiques, et fournit, en même temps, les préalables nécessaires pour une comparaison agénétique. Les comparaisons lexicographique et traductrice dégagent une richesse de données qui servent de base solide de recherche.

C'est à la comparaison structurelle, complétée par les trouvailles des comparaisons contrastive, interférentielle et, le cas échéant, évaluative, qu'il incombe de structurer le domaine de la comparaison bilingue intégrale et de mettre sur pied l'étude comparative phonique, graphique, grammaticale, lexicale et stylistico-textuelle des deux langues. Une telle structuration présuppose la sélection d'une conception linguistique adéquate et l'élaboration d'un système notionnel de l'étude comparative. Il y a donc pluralité de solutions théoriques. 
Quelle est, enfin, l'utilité de la comparaison bilingue ? Quels en sont les BUTS ? C'est d'abord de mieux saisir la structure de l'une ou de l'autre des deux langues, et sa complexité. C'est d'approfondir et de raffiner la connaissance et la compréhension des rapports entre les deux langues, surtout dans leur fonctionnement contemporain. C'est de se rendre compte des raisons profondes des difficultés que nous éprouvons en faisant face à une langue seconde, et qu'autrui éprouve en confrontant la nôtre.

Mais, il y a d'autres buts, qui dépassent le domaine linguistique. C'est, d'abord, de comprendre les aspects linguistiques des affinités et des conflits culturels, intellectuels, historiques entre les groupes de langue différente. C'est, en principe, d'élargir les horizons culturo-linguistiques au-delà de l'étroite perspective des unilingues. Le but est, encore, de voir les limites des interférences acceptables, aussi bien que la marge étroite de l'harmonisation possible entre les deux langues. C'est, surtout, d'apprendre la diversité des langues, la relativité des attitudes envers la langue maternelle et envers la langue seconde, et - ce faisant - d'apprendre la tolérance.

\section{BIBLIOGRAPHIE}

ARENS, Hans (1969) : Sprachwissenschaft. Der Gang ihrer Entwicklung van der Antike bis zur Gegenwart, 2, Auflage, Freiburg/München, Karl Alber.

BALLY, Charles (1950): Linguistique générale et linguistique française, Berne, Francke (1 ${ }^{\text {re }}$ éd. 1943).

DEROY, Louis (1956) : l'Emprunt linguistique, Paris, Les Belles lettres.

DIDEROT, Denis et Jean Le Rond D'ALEMBERT (1966) : Encyclopédie ou dictionnaire raisonné des arts et des métiers (éd. 1751-1780).

DUBOIS, Jean et al. (1973) : Dictionnaire de linguistique, Paris, Larousse.

ELLIS, Jeffrey (1966) : Towards a General Comparative Linguistics, The Hague, Mouton.

FISIAK, Jacek, Ed. (1981) : Contrastive Linguistics and the Language Teacher, Oxford, Pergamon Press. FOULQUIÉ, Paul (1969) : Dictionnaire de la langue philosophique, $2^{\mathrm{e}}$ éd., Paris, PUF.

GREIMAS, Algirdas Julien et Joseph COURTÉS (1979) : Sémiotique. Dictionnaire raisonné de la théorie du iangage, Paris, Hachette Université.

JAKOBSON, Roman (1963) : Essais de linguistique générale, Paris, Minuit.

JESPERSEN, Otto (1969) : Language, Its Nature, Development and Origin, London, Allen \& Unwin (1 ${ }^{\mathrm{rc}}$ éd., 1922).

KEIL, Heinrich, Ed. (1855-1880) : Grammatici Latini ex Recensione, Henrici Keilii, Leipzig, 7 vol.

KOCOUREK, Rostislav (1982) : la Langue française de la technique et de la science, Wiesbaden/Paris, Oscar Brandstetter/Documentation française.

KOCOUREK, Rostislav (1985) : "Terminologie et efficacité de la communication : critères linguistiques ", META, 30-2, pp. 119-128.

KUKENHEIM, Louis (1966) : Esquisse historique de la linguistique française et de ses rapports avec la linguistique générale, $2^{*}$ éd., Leiden, Universitaire Pers.

LALANDE, André (1951) : Vocabulaire technique et critique de la philosophie, $6^{e}$ éd., Paris, PUF.

MARTINET, André, éd. (1968) : le Langage, Encyclopédie de la Pléiade, Paris, Gallimard.

MARTINET, André (1969) : le Français sans fard, Paris, PUF.

MEILLET, Antoine (1952) : Linguistique historique et linguistique générale, t. II, Paris, Klincksieck.

MEILLET, Antoine (1969) : Introduction à l'étude comparative des langues indo-européennes, University of Alabama Press (1 $1^{\text {re }}$ éd.), 1903, Paris, Hachette.

MOUNIN, Georges (1967) : Histoire de la linguistique des origines au XXe siècle, Paris, PUF.

POTTIER, Bernard (1968) : la Typologie, Martinet éd., pp. 300-322.

ROBINS, R.H. (1967) : A Short History of Linguistics, London, Longmans.

ROBINS, R.H. (1976) : Brève histoire de la linguistique. De Platon à Chomsky, tr. de Robins (1967) par Mlaurice Borel, Paris, Seuil.

SAPIR, Edward (1921) : Language. An Introduction to the Study of Speech, New York, Harcourt, Brace \& World, s.d.

SAPIR, Edward (1953) : le Langage. Introduction à l'étude de la parole, tr. de Sapir (1921) par S.M. Guillemin, Paris, Payot.

TRNKA, Bohumil (1982) : Selected Papers in Structural Linguistics, Ed. V. Fried, Amsterdam, Mouton. VINAY, Jean-Paul et Jean DARBELNET (1968) : Stylistique comparée du français et de l'anglais, Montréal, Beauchemin (1re éd., 1958).

WEINREICH, Uriel (1968) : Unilinguisme et multilinguisme, Martinet éd. (1968), pp. 647-684.

WEI'NREICH, Uriel (1970) : Languages in Contact. Findings and Problems, The Hague, Mouton (1 éd., 1953) 